\title{
The Prognostic Role of the Surgical Approach and Adjuvant Therapy in Operable Mucosal Melanoma of the Head and Neck
}

\author{
GilJoon Lee* $\cdot$ Chung-Hwan Baek* $\cdot$ Na Yeon Choi $\cdot$ Man Ki Chung \\ Department of Otorhinolaryngology-Head and Neck Surgery, Samsung Medical Center, Sungkyunkwan University School of Medicine, \\ Seoul, Korea
}

Objectives. The aim of this study was to investigate the prognostic impact of the surgical approach and adjuvant treatment in operable malignant melanoma of head and neck (MMHN).

Methods. Retrospective reviews of 31 patients who underwent surgery-based treatment with curative intent, either by the endoscopic or external approach, for MMHN were performed to analyze recurrence patterns, salvage modalities, and oncological outcomes (disease-specific survival and disease-free survival).

Results. Overall recurrence rate was $61 \%(19 / 31)$. In stage III patients $(n=24), 50 \%(12 / 24)$ developed recurrences with a median recurrence-free period of 6.0 months, and 30\% (4/12) of them was successfully salvaged by reoperation with adjuvant radiotherapy. On the contrary, all stage IVA patients $(n=7)$ developed recurrences with a median recurrence-free period of 4.4 months. Distant metastasis was the most common pattern of failure and no patients were salvaged. Among variables, age and T classification, not the surgical approach, were significant prognosticators for disease-free survival and disease-specific survival. Adjuvant radiotherapy was associated with a lower rate of local failure, compared to surgery alone (hazard ratio, $0.02 ; 95 \%$ confidence interval, 0.06 to $0.75 ; P=0.02$ ). However, adjuvant systemic therapy was not effective in reducing the risk of failures for any pattern.

Conclusion. Our data suggested that meticulous surgical resection, either by the endoscopic or external approach, with adjuvant radiotherapy increases the local control rate in MMHN.

Keywords. Mucosal Melanoma; Head and Neck; General Surgery; Prognosis; Radiotherapy

\section{INTRODUCTION}

Mucosal melanoma of head and neck (MMHN) is an extremely rare disease entity, accounting for $1 \%$ to $3 \%$ of all malignant melanoma diagnoses and almost $6 \%$ of head and neck melano-

\footnotetext{
- Received January 11, 2016

Revised February 6, 2016

Accepted February 7, 2016

- Corresponding author: Man Ki Chung

Department of Otorhinolaryngology-Head and Neck Surgery, Samsung

Medical Center, Sungkyunkwan University School of Medicine, 81 Irwon-

ro, Gangnam-gu, Seoul 06351, Korea

Tel: +82-2-3410-3577, Fax: +82-2-3410-6987

E-mail: manki.chung@gmail.com

*The first two authors contributed equally to this study.
}

mas [1,2]. Common sites of involvement in the head and neck are the nasal cavity and the paranasal sinuses, accounting for up to $72 \%$ of patients $[3,4]$. Nonspecific symptoms and complex anatomic structures of the head and neck often delay early diagnosis of MMHN. Furthermore, due to high incidence of local recurrence and distant metastasis, MMHN is well known for poor prognosis with the 5-year overall survival (OS) ranging from $15 \%$ to $35 \%$ [5]. Although definitive guidelines for optimal management have not been established, proper surgical resection with a clear margin is accepted as the mainstay of treatment in operable MMHN [6,7].

Besides the recent revision of MMHN cancer staging in the 7th edition of the American Joint Committee on Cancer (AJCC) cancer staging manual, debatable issues regarding standard, on-

Copyright @ 2017 by Korean Society of Otorhinolaryngology-Head and Neck Surgery.

This is an open-access article distributed under the terms of the Creative Commons Attribution Non-Commercial License (http://creativecommons.org/licenses/by-nc/4.0)

which permits unrestricted non-commercial use, distribution, and reproduction in any medium, provided the original work is properly cited. 
Table 1. Patient profile

\begin{tabular}{|c|c|c|c|c|}
\hline Characteristic & All $(n=31)$ & Endoscopic approach $(n=16)$ & External approach $(n=15)$ & $P$-value \\
\hline Age (yr) & $56.0(50.0-71.0)$ & $61.0(52.2-71.7)$ & $53.0(44.0-71.0)$ & 0.15 \\
\hline Sex (male:female) & $18: 13$ & $10: 6$ & $8: 7$ & 0.67 \\
\hline Primary sites & & & & 0.006 \\
\hline Sinonasal & $25(81)$ & 16 & 9 & \\
\hline Oral & $5(16)$ & 0 & 5 & \\
\hline Oropharyngeal & $1(3)$ & 0 & 1 & \\
\hline T classification & & & & 0.03 \\
\hline T3 & $24(77)$ & 15 & 9 & \\
\hline $\mathrm{T} 4 \mathrm{a}$ & $7(23)$ & 1 & 6 & \\
\hline $\mathrm{T} 4 \mathrm{~b}$ & 0 & 0 & 0 & \\
\hline Staging & & & & 0.03 \\
\hline III & $24(77)$ & 15 & 9 & \\
\hline IVA & $7(23)$ & 1 & 6 & \\
\hline IVB & 0 & 0 & 0 & \\
\hline IVC & 0 & 0 & 0 & \\
\hline Treatment & & & & 0.55 \\
\hline OP only & $9(29)$ & 6 & 3 & \\
\hline $\mathrm{OP}+\mathrm{RT}$ & $13(42)$ & 7 & 6 & \\
\hline $\mathrm{OP}+$ Chemo/IFN & $7(23)$ & 2 & 5 & \\
\hline $\mathrm{OP}+\mathrm{RT}+\mathrm{Chemo} / \mathrm{FN}$ & $2(7)$ & 1 & 1 & \\
\hline Clear resection margin & & & & 0.63 \\
\hline Yes & $28(90)$ & 14 & 14 & \\
\hline No & $3(10)$ & 2 & 1 & \\
\hline Follow-up (mo) & $9.0 \pm 29.9$ & $10.0 \pm 2.1$ & $8.0 \pm 41.6$ & 0.21 \\
\hline Final status & & & & 0.009 \\
\hline No evidence of disease & $14(45)$ & 11 & 3 & \\
\hline Alive with disease & $3(10)$ & 2 & 1 & \\
\hline Death of disease & $13(34)$ & 3 & 10 & \\
\hline Died from other cause & $1(4)$ & 0 & 1 & \\
\hline
\end{tabular}

Values are presented as median (interquartile range), number (\%), or mean \pm standard deviation.

$\mathrm{OP}$, operation; RT, radiotherapy; Chemo, chemotherapy; IFN, interferon.

cologic care have been observed in localized MMHN, recently [8]. First, in light of advancements in optic technology, the incidence of endoscopic or endoscopy-assisted surgery, especially for sinonasal disease, is on the increase to ensure more precise resection as well as better cosmetic outcomes, compared to external approaches $[4,8,9]$. Second, the role of postsurgical, adjuvant treatment, such as postoperative radiotherapy (PORT) or systemic therapy, has been still controversial due to contradicting reports $[1,10,11]$.

\section{H I G G H L I G G H T S}

- This study showed that meticulous surgical resection followed by adjuvant radiotherapy increases the local control rate in mucosal melanoma of head and neck (MMHN).

- However, adjuvant systemic therapy (chemotherapy and interferon) was not effective in increasing disease control rate.

- Further studies for improving survival rate in MMHN are necessary.
The purpose of this study is to investigate the prognostic impact of different, surgical approaches (external vs. endoscopic) and postoperative, adjuvant treatments (PORT and/or systemic therapy) on oncological outcomes, measured as treatment failure patterns, disease-free survival (DFS), and disease-specific survival (DSS) in patients with stage III/IVA MMHN, who underwent surgery-based treatment with curative intent.

\section{MATERIALS AND METHODS}

\section{Patients}

From the cancer registry of Samsung Medical Center, 52 patients were treated for MMHN from 1999 to 2015. Inclusion criteria for the present study were as follows: (1) histopathological diagnosis of MMHN; (2) surgery-based, initial treatments with curative intent; and (3) integrity of clinical data. The 7th edition of the AJCC Cancer Staging Manual for MMHN was used in this study, and if patients were diagnosed and treated before the year 2009 when the 7th staging manual was released 
$(\mathrm{n}=7)$, restaging was performed based on preoperative physical and imaging studies as well as postoperative, pathological data [8]. Patients with melanoma that had an origin other than mucosa (eq., cutaneous), recurrent or inoperable cases (eq., presence of distant metastasis), and patients with incomplete data were excluded from the present study. A total of 31 patients were enrolled for the current study and retrospective review of their medical records was performed with regards to the demographic and clinicopathological data, as summarized in Table 1. Operability as well as the approach (either endoscopic or external) was determined on the basis of the location and the extent of the primary tumor, the feasibility of endoscopic instrumentation, and the necessity of post-ablative reconstruction. Subjects were analyzed and compared for the endpoints of the current study: (1) recurrence rates and patterns after initial treatments, (2) prognosticators for DFS/DSS, and (3) impact of adjuvant treatments on failure of any type.

\section{Statistical analysis}

Statistical analysis was performed using IBM SPSS ver. 23.0 (IBM Co., Armonk, NY, USA). Fisher exact test or Pearson chisquare analysis were used to evaluate the associations between the variables. Multivariate analysis was performed by logistic regression. Kaplan-Meier estimates and log-rank test were used to compare the variables against DFS and DSS. A Cox proportional hazard model was used to determine the influence of each variable on the survival plot. A two-sided $P<0.05$ was considered statistically significant.

\section{RESULTS}

Median age was 56.0 years old (interquartile range, 50.0 to 71.0 years old) and sex distribution (male:female) was 18:13. The most common primary site was sinonasal $(81 \%, 25 / 31)$, followed by oral cavity $(16 \%)$ and oropharyngeal $(3 \%)$. No patient showed nodal disease at initial presentation, $77 \%$ of patients (24/31) were classified as T3N0 (stage III), and the remaining patients (7/31) were classified as T4aN0 (stage IVA). Sixteen patients (15 T3 and 1 T4a) were treated with the endoscopic approach only, and the other 15 patients (9T3 and 6 $44 a$ ) received the external approach for curative surgery. As expected, the external approach was preferred for patients with stage IVA (6/7, $86 \%)$, compared to patients with stage III $(9 / 24,38 \%)$ with a statistical difference $(P=0.03)$. By subgroup analyses according to the surgical approach, mean age, sex distribution, and primary sites were balanced between the two groups. Regardless of the surgical approach, operation followed by PORT was the treatment of choice for our cohort $(42 \%)$. The number of patients treated with operation alone and with operation followed by systemic therapy only (chemotherapeutic agents or high-dose interferon) was similar ( $29 \%$ and $23 \%$, respectively). With a median follow-up of 9.0 months, $45 \%$ of patients remained free of disease, $34 \%$ of patients died of disease, and $10 \%$ of patients were still alive with disease.

\section{Recurrence rates and patterns}

The total recurrence rate for our cohort was $61 \%$ (19/31). Spe-

Table 2. Recurrence patterns and salvage outcomes

\begin{tabular}{|c|c|c|c|c|c|c|}
\hline No. & Initial stage & 1st treatment & Recurrence-free period (mo) & Recurrence type & Salvage & Final status \\
\hline 1 & III & OP & 45 & L & $\mathrm{OP}+\mathrm{RT}$ & NED \\
\hline 2 & & $\mathrm{OP}+\mathrm{IFN}$ & 6 & L & $\mathrm{OP}+\mathrm{RT}$ & NED \\
\hline 3 & & $\mathrm{OP}+\mathrm{IFN}$ & 13 & L & $\mathrm{OP}+\mathrm{RT}$ & AWD \\
\hline 4 & & OP & 19 & L & $\mathrm{OP}+\mathrm{RT}$ & NED \\
\hline 5 & & $\mathrm{OP}$ & 6 & L & $\mathrm{OP}+\mathrm{RT}$ & NED \\
\hline 6 & & $\mathrm{OP}$ & 6 & $L, R$ & Refused & DOD \\
\hline 7 & & $\mathrm{OP}+\mathrm{RT}$ & 1 & $L, D$ & Refused & DOD \\
\hline 8 & & $\mathrm{OP}+\mathrm{IFN}$ & 4 & $\mathrm{R}$ & $\mathrm{OP}+\mathrm{RT}$ & DOD \\
\hline 9 & & $\mathrm{OP}+\mathrm{RT}+\mathrm{IFN}$ & 12 & $\mathrm{D}$ & $\mathrm{OP}+$ Chemo & DOD \\
\hline 10 & & $\mathrm{OP}+\mathrm{RT}$ & 10 & $\mathrm{D}$ & Chemo & AWD \\
\hline 11 & & $\mathrm{OP}+\mathrm{RT}$ & 5 & $D$ & Chemo & DOD \\
\hline 12 & & $\mathrm{OP}$ & 15 & $\mathrm{D}$ & Refused & AWD \\
\hline 13 & IVA & $\mathrm{OP}+\mathrm{IFN}$ & 4 & L & $\mathrm{RT}$ & DOD \\
\hline 14 & & $\mathrm{OP}+\mathrm{RT}$ & 8 & $L, R$ & $\mathrm{OP}+\mathrm{RT}$ & DOD \\
\hline 15 & & $\mathrm{OP}+\mathrm{Chemo}$ & 2 & $L, D$ & Chemo & DOD \\
\hline 16 & & $\mathrm{OP}+\mathrm{RT}$ & 2 & $R, D$ & Refused & DOD \\
\hline 17 & & $\mathrm{OP}+\mathrm{RT}$ & 2 & $\mathrm{D}$ & Chemo & DOD \\
\hline 18 & & $\mathrm{OP}+\mathrm{RT}$ & 6 & D & Refused & DOD \\
\hline 19 & & $\mathrm{OP}+\mathrm{RT}$ & 7 & D & Chemo & DOD \\
\hline
\end{tabular}

OP, operation; L, local recurrence; RT, radiotherapy; NED, no evidence of disease; IFN, interferon; AWD, alive with disease; R, locoregional recurrence; $\mathrm{DOD}$, death of disease; $\mathrm{D}$, distant metastasis; Chemo, chemotherapy. 
cifically, local recurrence developed in 19\% (6/31), regional recurrence in $3 \%$ (1/31), distant metastasis in $23 \%$ (7/31, most commonly in the lung), and recurrences developed in multiple sites in 16\% (5/31, 2 loco-regional, 2 loco-distant, and 1 regional-distant). Recurrence patterns and related salvage treatments for the cohort are described in Table 2.

The median recurrence-free period for local recurrence in 6 patients ( 5 in stage III and 1 in stage IVA) was 6.0 months. As initial treatments for those patients, 3 patients were treated by surgery only. Systemic high dose interferon was administered after surgery in the other 3 patients because the benefit of adjuvant radiotherapy was considered negligible as most of the $\mathrm{T}$ classification was T1-2 (except 1 T3) according to the version of AJCC staging released at the time. After recurrence, re-operation with PORT was the salvage treatment of choice, except in 1 patient who underwent radiotherapy only due to inoperable recurrent disease. Among the local recurrence cases, 4 patients (4/6, 67\%) were successfully salvaged, 1 patient remained alive with disease, and the last patient died of disease (from initial stage IVA). For 4 regionally recurred patients (1 regional, 2 loco-regional, and 1 regional-distant), reoperation with PORT was performed in 2 patients who had either regional or loco-regional recurrence.

However, all failed to be salvaged. The other 2 patients refused further treatments. Similarly, every patient that developed distant metastasis died from disease despite palliative systemic therapy, with the exception of 1 patient who was still alive with disease. When the recurrence pattern was analyzed according to the initial stage, local recurrence was most common in stage III $(5 / 12,42 \%)$, and the success rate of salvage was 33\% (4/12). On the contrary, distant metastasis or multiple recurrences was the most common pattern in stage IVA $(6 / 7,86 \%)$, and no patients were salvaged after recurrences.

The results showed that the 5-year local recurrence-free survival was $43 \%$, regional recurrence-free survival was $84 \%$, and distant metastasis-free survival was 59\%. DFS, DSS, and OS were $33 \%, 52 \%$, and $48 \%$, respectively.

\section{Prognosticators for DFS and DSS}

According to the results of the Kaplan-Meier estimate, the median DFS of the entire cohort was $12.0 \pm 29.9$ months $(95 \%$ confidence interval [CI], 6.3 to 17.6 months) and the median DSS was $9.0 \pm 29.9$ months (95\% CI, 4.2 to 15.2 months). The median DFS of patients with stage IVA was significantly shorter than that of patients with stage III (stage III, 11 months; 95\% CI, 5.7 to 16.4 months vs. stage IVA, 4 months; $95 \%$ CI, 2.5 to 6.3 months; log-rank $P<0.001)$. Similarly, the median DSS of stage IVA patients was 4.0 months (95\% CI, 2.3 to 6.1 months), which was significantly shorter than that of stage III patients with a median of 12 months (95\% CI, 6.4 to 17.4 months; logrank $P<0.001)$. Among the various clinicopathological variables available (age, sex, primary site, $\mathrm{T}$ classification, type of surgical approach, surgical margin status, and adjuvant modality), prognosticators for survival estimates were evaluated by univariate and multivariate analysis (Table 3). The results showed that earlier age and advanced $\mathrm{T}$ classification were significant prognosticators for worse DFS (age, hazard ratio [HR], 0.26; 95\% CI, 0.09 to $0.79 ; P=0.01$; T classification, $\mathrm{HR}, 10.8 ; 95 \% \mathrm{CI}, 2.7$ to 42.7 ; $P=0.001$ ) as well as worse DSS (age, HR, 0.26; 95\% CI, 0.09 to $0.89 ; P=0.04$; T classification, HR, $14.4 ; 95 \% \mathrm{CI}, 2.7$ to 42.7 ; $P=0.002)$. The surgical approach or the application of adjuvant treatments did not show any prognostic impact on survival in operated MMHN.

\section{Impact of adjuvant treatments on failure of any type}

Correlation between the types of adjuvant treatments and failure patterns was investigated as shown in Table 4. By the Cox proportional hazard model, the application of PORT was associated with reduced risk of local recurrence. Specifically, contrary to a

Table 3. Analyses of prognostic factors for DFS and DSS

\begin{tabular}{|c|c|c|c|c|}
\hline \multirow{2}{*}{ Variable (reference) } & \multicolumn{2}{|c|}{ DFS } & \multicolumn{2}{|c|}{ DSS } \\
\hline & $\mathrm{HR}(95 \% \mathrm{Cl})$ & $P$-value ${ }^{\text {a) }}$ & $\operatorname{HR}(95 \% \mathrm{Cl})$ & $P$-value \\
\hline \multicolumn{5}{|l|}{ Univariate } \\
\hline Age ( $\leq 56$ yr vs. $>56$ yr) & $0.35(0.13-0.90)$ & 0.03 & $0.21(0.05-0.71)$ & 0.02 \\
\hline Sex (male vs. female) & $0.67(0.25-1.78)$ & 0.43 & $1.00(0.31-2.89)$ & 0.78 \\
\hline Site (sinonasal vs. other) & $1.14(0.37-3.46)$ & 0.81 & $2.33(0.68-7.99)$ & 0.17 \\
\hline T classification (T3 vs. T4) & $6.59(2.16-20.1)$ & 0.001 & $13.2(3.3-53.1)$ & $<0.001$ \\
\hline Surgical approach (endoscopic vs. external) & $1.36(0.53-3.45)$ & 0.51 & $3.41(0.9-12.6)$ & 0.06 \\
\hline Clear resection margin (no vs. yes) & $0.55(0.07-4.24)$ & 0.56 & $0.79(0.10-6.32)$ & 0.82 \\
\hline Treatment modality (no adjuvant vs. adjuvant) & $1.96(0.69-5.58)$ & 0.20 & $7.09(0.90-55.6)$ & 0.06 \\
\hline \multicolumn{5}{|l|}{ Multivariate } \\
\hline Age ( $\leq 56$ yr vs. $>56$ yr $)$ & $0.26(0.09-0.79)$ & 0.01 & $0.26(0.09-0.89)$ & 0.04 \\
\hline T classification (T3 vs. T4) & $10.8(2.7-42.7)$ & 0.001 & $14.4(2.7-42.7)$ & 0.002 \\
\hline Surgical approach (endoscopic vs. external) & $0.35(0.10-1.17)$ & 0.09 & $0.75(0.16-1.17)$ & 0.72 \\
\hline
\end{tabular}

DFS, disease free survival; DSS, disease specific survival; HR, hazard ratio; $\mathrm{Cl}$, confidence interval.

a)Cox proportional hazard model. 
Table 4. Correlations between adjuvant treatments and failure types

\begin{tabular}{|c|c|c|c|c|c|c|c|c|c|}
\hline \multirow{2}{*}{ Adjuvant } & \multicolumn{3}{|c|}{ Local failure } & \multicolumn{3}{|c|}{ Regional failure } & \multicolumn{3}{|c|}{ Distance failure } \\
\hline & Event (\%) & $\mathrm{HR}(95 \% \mathrm{Cl})$ & $P$-value & Event (\%) & $\mathrm{HR}(95 \% \mathrm{Cl})$ & $P$-value & Event (\%) & $\mathrm{HR}(95 \% \mathrm{Cl})$ & $P$-value \\
\hline None & $4 / 9(44)$ & 1 & & $1 / 9(11)$ & 1 & & $1 / 9(11)$ & 1 & \\
\hline RT & 2/13(15) & $0.02(0.06-0.75)$ & 0.02 & 2/13 (15) & $0.66(0.03-12.2)$ & 0.78 & $7 / 13(54)$ & $0.88(0.14-5.47)$ & 0.89 \\
\hline Systemic & $4 / 7(57)$ & $0.60(0.08-4.40)$ & 0.61 & $1 / 7(14)$ & $1.33(0.06-25.9)$ & 0.84 & $1 / 7(14)$ & $0.33(0.02-4.18)$ & 0.39 \\
\hline $\mathrm{RT}+$ systemic & $0 / 2(0)$ & $0.80(0.03-17.1)$ & 0.88 & $0 / 2(0)$ & 0 & 0 & $1 / 2(50)$ & $2.00(0.09-44.3)$ & 0.66 \\
\hline
\end{tabular}

$\mathrm{HR}$, hazard ratio; $\mathrm{Cl}$, confidence interval; RT, radiotherapy; Systemic, systemic therapy (chemotherapy or interferon).

$44 \%$ local recurrence rate in patients with no adjuvant therapy, patients with PORT showed a lower local recurrence rate of $15 \%$ with a HR of $0.02(95 \% \mathrm{CI}, 0.06$ to $0.75 ; P=0.02)$. When subgroup analysis was performed to adjust for the cancer stage, patients with PORT showed better 2-year local recurrence-free survival than patients without PORT even within stage III $(90.0 \%$ vs. $50.0 \%$, log-rank $P=0.03)$. On the contrary, PORT showed no association with better or worse survival estimates in regional recurrence or distant metastasis in stage III patients, as well as in stage IV patients. Other adjuvant modalities, such as systemic therapy or PORT with systemic therapy, did not show any preventive impact to reduce the risk of any type of failure.

\section{DISCUSSION}

The present study aimed to investigate the impact of the surgical approach and adjuvant treatment modalities on oncologic outcomes in operable, stage III/IVA MMHN.

Because MMHN is known to aggressively invade adjacent tissue and occur frequently with skipped lesions, it is necessary to obtain wide surgical resection with clear margin, which is shown to be an important prognostic factor $[12,13]$. However, complex anatomic structures such as the nasal cavity and the paranasal sinuses, sites commonly involved in MMHN, could impede meticulous resection of tumor even with open, external approaches (eq., maxillectomy), often resulting in unsatisfactory aesthetic appearance and three dimensional defects, requiring reconstruction. That is why the endoscopic technique has been gaining popularity for dealing with various tumors in the sinonasal region with acceptable outcomes [12]. Among the sparse clinical studies conducted on endoscopic surgery for MMHN, it was reported that endoscopically resected patients $(27 \%, 31$ out of 115 with sinonasal malignant melanoma) had a significant OS advantage for up to 5 years (endoscopic $45.6 \%$ vs. open minor $24 \%$ vs. open major $10 \%$ ) [3]. The results suggested that endoscopic techniques may be employed with at least similar outcomes and minimal morbidity. Another study involving large case series of sinonasal malignant melanoma demonstrated that among 70 out of 133 patients that underwent endoscopic or endoscopy-assisted surgery, $37.1 \%$ experienced recurrences, which was significantly lower than patients treated with the external approach only [4]. Also, it was shown that the endoscopic approach was associated with better survival, compared to the external approach group with statistical significance (HR, 1.702). The present study is different from previous reports in that pure endoscopic surgery, not endoscopy-assisted surgery, was performed to resect MMHN in all 16 cases, and clear information on the AJCC stage was described to avoid potential bias from a preference for endoscopic techniques in the early stages of cancer. Notably, the endoscopic approach was adopted more frequently in stage III $(15 / 24,62.5 \%)$, than in stage IVA $(1 / 7$, $14.2 \%)$. It is also interesting that endoscopic approaches replaced open approaches in the latter series of our cohort $(13 / 16$, $81.2 \%$ performed after the year 2012), reflecting the trend toward minimally invasive techniques.

Although the present study is consistent with previous reports in that the endoscopic approach was sufficient to provide adequate resection margins as well as minimal aesthetic morbidity, clear margins could not be obtained in 2 cases with endoscopic approaches. In one case, the melanoma lesion directly invaded the Eustachian tube, and complete resection was not feasible even under endoscopic view. In the other case of sinonasal melanoma, clear margin was obtained in frozen pathology during surgery, but melanocytic nests were revealed in permanent pathology.

Recurrence control rates in the present study showed similarities to previous studies, in which 5-year local control rates ranged from $40 \%$ to $79 \%$ (present study, $43 \%$ ), regional control rates ranged from $71 \%$ to $83 \%(84 \%)$, and distant metastasis control rates ranged from $17 \%$ to $49 \%$ (59\%) [6,14-18]. Fiveyear DFS and OS were also in close agreement with previous reports, where DFS ranged from $4 \%$ to $40 \%$ (present study, $25 \%$ ), and OS ranged from $20 \%$ to $48 \%$ (48\%). However, it is not in accord with previous reports in that endoscopic approaches were not associated with better recurrence rates or survival gain, when the stage and other variables were adjusted by the Cox proportional hazard model in our study. Compared with other studies, there was no difference in the prognosis of MMHN (recurrence rate and survival rate) $[9,19,20]$.

The role of adjuvant treatment needs to be determined to improve the oncologic outcomes of MMHN. It has been shown by recent meta-analysis that postoperative radiotherapy improves loco-regional recurrence of MMHN (HR, 0.36; 95\% CI, 0.22 to 
$0.60 ; P=0.000)$, independent of OS [11]. The present study coincided with previous study in that PORT showed a significant association with reduced risk of local recurrence compared to no adjuvant treatment (HR, $0.02 ; P=0.02)$. Interestingly, the preventive impact of PORT for local control was evident in stage III patients, not stage IV, as patients with PORT showed better local recurrence-free survival than patients without PORT in stage III by the log-rank test. However, PORT did not show any association with survival benefits in this study, as with other reports $[1,10,11]$.

After recurrence, salvage was successful only in patients with local recurrence treated by reoperation followed by PORT, giving a total salvage success rate of $2 \%(4 / 19)$ in this study. Contrary to patients with local recurrence, every regionally recurred case $(n=4)$ failed to be salvaged, which could be attributable partly to the patients' refusal to accept further treatments (2/4), and more importantly, to combined sites of recurrences (local or distant sites). It is also disappointing that the initial stage for all salvaged patients was confined to stage III, and that distant metastasis was the most common pattern of failure in stage IVA patients, which could not be salvaged by any form of treatment in our cohort. Among patients with systemic therapy $(n=9)$, highdose interferon was the most preferred regimen combined with or without PORT, except for 1 case treated with a chemotherapeutic agent in the present study $(8 / 9,89 \% ; 3$ cases in stage III and 5 cases in stage IVA). During follow-up, loco-regional recurrence developed in 4 cases ( 3 local and 1 regional), all of whom did not take PORT, and distant metastasis occurred in 1 case. Although it is well known that efficient control of hematogenous spread in MMHN is critical to improve the high rates of distant failures, the application of systemic therapy did not show any efficacy in preventing failure of any type in this study.Therefore, outcomes with novel systemic therapies, such as tyrosinekinase inhibitor or immunotherapeutic agents, need to be investigated in the form of multicenter, prospective studies [21,22].

Major limitations of the present study are (1) the retrospective nature of the study in a single institute and (2) the small number of enrolled patients. These problems may stem from the rarity of the disease, requiring a long period to accumulate a sufficient number of cases. It is appreciable that the period for the present study (from 1999 to 2015) was relatively shorter than for previous studies, supporting the idea that the treatment protocols and related outcomes of the current study are less likely to be biased by different phases of study $[6,20,23]$.

In conclusion, adequate surgical resection must be implemented either with the open or endoscopic approach and combined to obtain wide and clear surgical margins in operable MMHN. PORT, also, should be incorporated with postsurgical, adjuvant modality because it has been proven to be effective in reducing the risk of local and/or loco-regional recurrences. Systemic therapy requires further investigations and trials to improve survival in MMHN.

\section{CONFLICT OF INTEREST}

No potential conflict of interest relevant to this article was reported.

\section{REFERENCES}

1. Christopherson K, Malyapa RS, Werning JW, Morris CG, Kirwan J, Mendenhall WM. Radiation therapy for mucosal melanoma of the head and neck. Am J Clin Oncol. 2015 Feb;38(1):87-9.

2. Siegel R, Naishadham D, Jemal A. Cancer statistics, 2013. CA Cancer J Clin. 2013 Jan;63(1):11-30.

3. LundVJ, Chisholm EJ, Howard DJ,WeiWI. Sinonasal malignant melanoma: an analysis of 115 cases assessing outcomes of surgery, postoperative radiotherapy and endoscopic resection. Rhinology. 2012 Jun;50(2):203-10.

4. Won TB, Choi KY, Rhee CS, Jin HR, Yi JS, Dhong HJ, et al. Treatment outcomes of sinonasal malignant melanoma: a Korean multicenter study. Int Forum Allergy Rhinol. 2015 Oct;5(10):950-9.

5. Gru AA, Becker N, Dehner LP, Pfeifer JD. Mucosal melanoma: correlation of clinicopathologic, prognostic, and molecular features. Melanoma Res. 2014 Aug;24(4):360-70.

6. Chan RC, Chan JY, Wei WI. Mucosal melanoma of the head and neck: 32-year experience in a tertiary referral hospital. Laryngoscope. 2012 Dec;122(12):2749-53.

7. Troussier I, Baglin AC, Marcy PY, Even C, Moya-Plana A, Krengli M, et al. Mucosal melanomas of the head and neck: state of the art and current controversies. Bull Cancer. 2015 Jun;102(6):559-67.

8. Edge SB, Byrd DR, Compton CC, Fritz AG, Greene FL, Trotti A. AJCC cancer staging manual. 7th ed. New York (NY): Springer; 2009.

9. Gal TJ, Silver N, Huang B. Demographics and treatment trends in sinonasal mucosal melanoma. Laryngoscope. 2011 Sep;121(9):202633.

10. Benlyazid A, Thariat J,Temam S, Malard O, Florescu C, Choussy O, et al. Postoperative radiotherapy in head and neck mucosal melanoma: a GETTEC study. Arch Otolaryngol Head Neck Surg. 2010 Dec; 136(12):1219-25

11. Wushou A, Hou J, Zhao YJ, Miao XC. Postoperative adjuvant radiotherapy improves loco-regional recurrence of head and neck mucosal melanoma. J Craniomaxillofac Surg. 2015 May;43(4):553-8.

12. Nicolai P, Battaglia P, Bignami M, BolzoniVillaretA, Delu G, KhraisT, et al. Endoscopic surgery for malignant tumors of the sinonasal tract and adjacent skull base: a 10-year experience. Am J Rhinol. 2008 May-Jun;22(3):308-16.

13. Penel N, Mallet Y, Mirabel X,Van JT, Lefebvre JL. Primary mucosal melanoma of head and neck: prognostic value of clear margins. Laryngoscope. 2006 Jun;116(6):993-5.

14. Stern SJ, Guillamondegui OM. Mucosal melanoma of the head and neck. Head Neck. 1991 Jan-Feb;13(1):22-7.

15. Guzzo M, Grandi C, Licitra L, Podrecca S, Cascinelli N, Molinari R. Mucosal malignant melanoma of head and neck: forty-eight cases treated at Istituto Nazionale Tumori of Milan. Eur J Surg Oncol. 1993 Aug;19(4):316-9.

16. Patel SG, Prasad ML, Escrig M, Singh B, Shaha AR, Kraus DH, et al. Primary mucosal malignant melanoma of the head and neck. Head Neck. 2002 Mar;24(3):247-57.

17. Temam S, Mamelle G, Marandas P, Wibault P, Avril MF, Janot F, et al. Postoperative radiotherapy for primary mucosal melanoma of the head and neck. Cancer. 2005 Jan;103(2):313-9.

18. Wagner M, Morris CG, Werning JW, MendenhallWM. Mucosal mel- 
anoma of the head and neck. Am J Clin Oncol. 2008 Feb;31(1):438.

19. Koivunen P, Bäck L, Pukkila M, Laranne J, Kinnunen I, Grenman R, et al. Accuracy of the current TNM classification in predicting survival in patients with sinonasal mucosal melanoma. Laryngoscope. 2012 Aug;122(8):1734-8.

20. Sun CZ, Li QL, Hu ZD, Jiang YE, Song M, Yang AK. Treatment and prognosis in sinonasal mucosal melanoma: a retrospective analysis of 65 patients from a single cancer center. Head Neck. 2014 May;36(5): 675-81.
21. Hodi FS, O’Day SJ, McDermott DF, Weber RW, Sosman JA, Haanen $\mathrm{JB}$, et al. Improved survival with ipilimumab in patients with metastatic melanoma. N Engl J Med. 2010 Aug;363(8):711-23.

22. Guo J, Si L, Kong Y, Flaherty KT, Xu X, Zhu Y, et al. Phase II, openlabel, single-arm trial of imatinib mesylate in patients with metastatic melanoma harboring c-Kit mutation or amplification. J Clin Oncol. 2011 Jul;29(21):2904-9.

23. Huang SF, Liao CT, Kan CR, Chen IH. Primary mucosal melanoma of the nasal cavity and paranasal sinuses: 12 years of experience. J Otolaryngol. 2007 Apr;36(2):124-9. 